\title{
Universal reference method for real-time PCR gene expression analysis of preimplantation embryos
}

\author{
Neil Ivan Bower ${ }^{1}$, Ralf Joachim Moser ${ }^{1}$, Jonathan Robert Hill2, and Sigrid Arabella Lehnert ${ }^{1}$
}

BioTechniques 42:199-206 (February 2007)

doi $10.2144 / 000112314$

\begin{abstract}
Real-time PCR expression profiling in individual preimplantation embryos poses two main challenges. First, the amount of RNA from blastocysts (between 100 and 200 cells) is too small to quantify, and secondly, a reference gene with stable expression across preimplantation embryos produced by different reproductive technologies is required. We have developed a method using RNA and DNA spikes that allows for accurate normalization of gene expression without the use of an internal housekeeping gene in preimplantation blastocysts. Prior to the simultaneous extraction of RNA and DNA, plant-specific RNA and DNA spikes are added to the tissue. After synthesis of cDNA, target gene transcript and the exogenous RNA spike are measured using real-time PCR. To account for differences in the number of cells in each sample, the genomic gene copies of 18S-DNA are measured by quantitative PCR and normalized to the DNA spike. While the DNA spike accounts for extraction efficiency, the $18 S$ genomic target indicates the number of cells prior to extraction. The values obtained from normalizing the target gene to the RNA spike can be adjusted for cell number, allowing the RNA spike to be used as reference gene. This universal reference approach allows the use of an exogenous spike as a pseudo-housekeeping gene for normalization of gene expression data.
\end{abstract}

\section{INTRODUCTION}

Real-time PCR is a powerful tool routinely used in gene expression analysis and quantification (1). To control for experimental and technical variation, appropriate normalization strategies have to be implemented. The most popular quantification strategy among other approaches is typically the normalization of the expression level of a particular target gene to the expression level of an internal housekeeping or reference gene (2-4). By normalizing the expression of a target gene to that of a reference gene, differences in the quality and quantity of starting RNA and in efficiencies of the reverse transcription reaction between samples can be accounted for. This allows the direct comparison of normalized expression values between samples.

For each new experimental system (new tissue samples and treatments), the various reference genes available need to be assessed to determine the most stable housekeeping gene (5). However, a stable housekeeper may not be available in certain experimental situations. For instance, the comparison of somatic cell nuclear transfer (SCNT) embryos with those produced by artificial insemination (AI) or in vitro fertilization (IVF) is confounded by a number of issues. SCNT embryos have been shown to have aberrant gene expression when compared with IVF embryos (6-8). Genes that have been used as housekeepers [e.g., glyeraldehyde-3-phosphate dehydrogenase $(G A P D H)$, actin $\beta(A C T B)]$ in the past may therefore be unsuitable as reference genes when comparing nuclear transfer (NT) and IVF embryos. In addition, controversial results on the gene expression stability of the histone $\mathrm{H} 2 \mathrm{a}(H 2 a-615)$ gene and its use as internal reference gene in preimplantation embryos are present in the literature $(9,10)$.
The study and quantification of gene expression in single preimplantation embryos is further complicated by the small amounts of starting material. The advancement and refinement of the Eberwine technique (11) to amplify RNA in a linear fashion, in combination with real-time PCR, has overcome these limitations but introduces a number of other issues and different sources of variation. The quality (integrity) and quantity (efficiency) of the resulting RNA/cDNA is dependent on many processes such as the extraction and amplification of the RNA, the reverse transcription reaction, and the amplification efficiency of the PCR.

To account for some of the problems listed above, researchers have used exogenously added spike-in RNAs, which can be used as an exogenous reference gene (12-14). These spikes are usually added to the samples during cDNA synthesis and therefore rely heavily on the accurate quantification 


\section{Research Reports}

of the RNA samples. As this exogenous spike is added at the end point in the process, it does not account for the integrity of the RNA sample or the reliability of any technical processing that has previously occurred. Addition of an exogenous reference RNA at the beginning of the entire process could account for any of these potential problems. Two reports describe the use of a commercially available messenger RNA (mRNA) as an exogenous reference for target gene normalization that was spiked into cell culture or tissue samples during the extraction process with only approximate or no knowledge of the starting material $(15,16)$. Yet, in order to use a spike-in RNA as a potential pseudo-housekeeping gene in small samples, the amount of starting material relative to the spike needs to be addressed. This is especially crucial if the amount of cells in the starting material is ranging from 100 to 300 cells, which would profoundly affect the spike-to-RNA ratio after extraction. Determining the number of cells in a given sample would allow the target gene to be normalized to the spike and this value subsequently adjusted for cell number. This would enable the spike to be used as an internal reference gene for the normalization of gene expression data. A current method to count cells in embryos involves the staining of the DNA in the nucleus and the assessment of the cell number under the microscope $(17,18)$. However, the time requirement and the fluorescent dye treatment are likely to have an effect on the transcriptome of these samples and are compromising the ultimate goal to measure gene expression in small biological material. Kanno et al. (19) report the use of Molecular Probes' PicoGreen ${ }^{\circledR}$ dye (Invitrogen Australia, Mount Waverley, VIC, Australia) to determine the number of cells in starting material that can then be used to calculate an appropriate amount of spike input for gene normalization. Using this method, the smallest sample size producing reliable data are approximately 5000 cells.

This paper proposes a novel, highly reliable, and universal application of a plant-specific transcript RNA (ptRNA) containing a poly(A) tail and
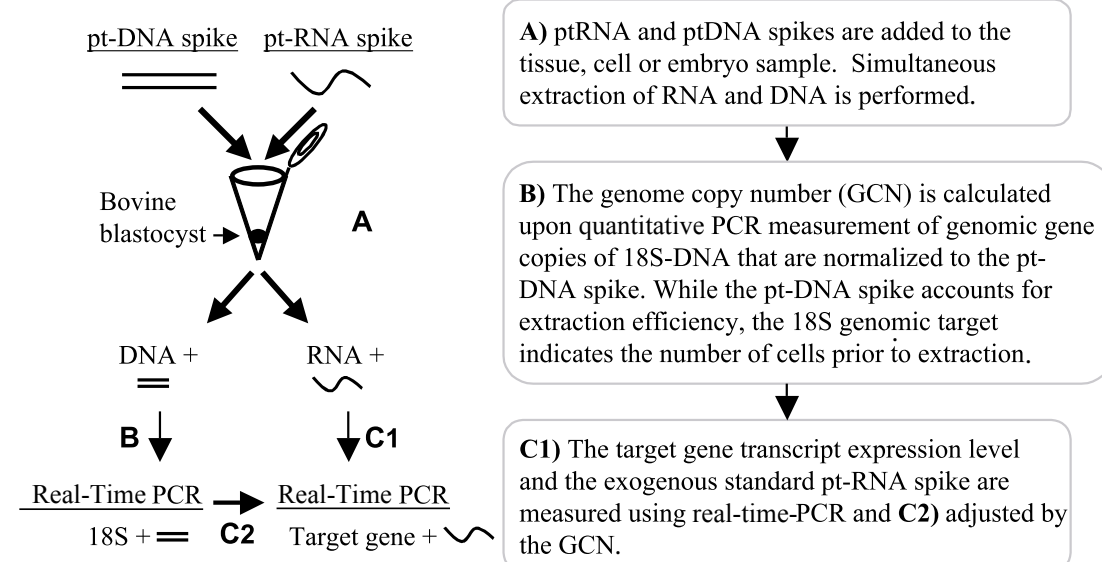

Figure 1. The Universal Reference Gene (URG) approach. The approach comprises three major steps, which will allow for the use of an external reference gene (spike-in control) to be used as a pseudo-housekeeping gene in small samples. pt-RNA, plant-specific transcript RNA; pt-DNA, PCR product corresponding to pt-RNA.

its corresponding PCR product (ptDNA) as exogenous spikes to measure nucleic acid extraction efficiencies and integrities and ultimately quantifying mRNA transcript expression levels during real-time PCR. This method has been termed Universal Reference Gene (URG) and comprises three steps, which are depicted in Figure 1.

This technique has the potential to be used as a universal reference gene assay to quantify gene expression in situations in which no stable reference gene is available and the sample size is small.

\section{MATERIALS AND METHODS}

\section{Preparation of Bovine Fibroblast Cells}

Bovine fibroblast cells were cultured in Dulbecco's modified Eagle's medium (DMEM) with $10 \%$ fetal bovine serum and $2 \%$ antibiotic-antimycotic solution (Invitrogen Australia) as described by Tan et al. (20). Once the cells reached confluence, they were trypsinized and resuspended. The cell concentration was calculated by averaging 10 cell counts using a $\mathrm{Z}^{\mathrm{TM}}$ Coulter Counter ${ }^{\circledR}$ (Beckman Coulter Australia, Gladesville, NSW, Australia) and adjusted to 20 cells $/ \mu \mathrm{L}$. Aliquots of cells were placed into tubes and centrifuged for $5 \mathrm{~min}$ at $800 \times \mathrm{g}$. The supernatant was completely removed, and the cells were snap-frozen in liquid nitrogen.

\section{AI and NT Embryo Production}

In vivo produced bovine embryos were recovered nonsurgically 7 days following insemination of superovulated cows (21). Briefly, cows were induced to superovulate using a commercial follicle stimulating hormone preparation (Folltropin $\left.{ }^{\circledR}\right)$ and a progesterone implant (Bioniche Animal Health, Armidale, NSW, Australia) and then were artificially inseminated. Embryos were recovered by uterine lavage and then graded for stage and quality.

NT bovine blastocysts were generated by transfer of fibroblast cells into enucleated oocytes (22). Oocytes were derived from ovaries sourced from abattoir cattle, and the fibroblasts were from cell culture. Fibroblast cells were derived from explant cultures of skin sections and were cultured for 2-4 passages prior to use as donor cells for NT. Embryos were cultured in a derivative of synthetic oviductal fluid serum-free medium (Cook Scientific, Brisbane, QLD, Australia) in a $6 \%$ $\mathrm{CO}_{2}, 5 \% \mathrm{O}_{2}, 89 \% \quad \mathrm{~N}_{2}$ atmosphere. Individual embryos were snap-frozen and stored at $-80^{\circ} \mathrm{C}$. All embryos used in this experiment were graded for stage and quality according to International Embryo Transfer Society (IETS) guidelines (23), and only grade 1 or 2 embryos (24) were used. 


\section{Research Reports}

\section{Production of Plant PCR Product (pt-DNA) and Corresponding Transcript (pt-RNA)}

A sugarcane cDNA clone (GenBank ${ }^{\circledR}$ accession no. CO373127), encoding a plant-specific protein (auxin response factor-like) that had no homology to bovine sequences as assessed by Basic Local Alignment Search Tool (BLAST) searches, was amplified using M13 forward and reverse primers from a phage stock. The resulting PCR product was sequenced, and its origin and size of the poly(A) tail (approximately 25 nucleotides) confirmed using BigDye ${ }^{\circledR}$ terminator chemistry 3.1 (Applied Biosystems, Foster City, CA, USA). Subsequently, it was in vitro transcribed using the MAXIscript ${ }^{\circledR}$ T7/T3 kit (Ambion, Austin, TX, USA) using the T7 RNA polymerase to yield the corresponding RNA transcript. The pt-RNA sample was DNasetreated, phenol/chloroform-extracted, and ethanol-precipitated. To produce the pt-DNA spike, the PCR product was used as a target in a PCR using M13 reverse primer in combination with a primer (ARF-G spike $1 \mathrm{R}$ 5'-CATCTGATCCATGTACTTCT CA-3') to yield a shorter fragment minus the poly(A) sequence. The ptDNA product was cleaned up using a QIAquick ${ }^{\circledR}$ PCR purification kit (Qiagen, Valencia, CA, USA). Spike concentrations were measured spectrophotometrically, and the amounts added to the samples were determined by real-time PCR. The amount of ptRNA spike added to each biological sample was calculated to equal a cycle threshold $\left(\mathrm{C}_{\mathrm{T}}\right)$ number of approximately 20 in the final real-time PCR measurement. Approximately 1000 times less pt-RNA spike was added to blastocyst samples to account for two rounds of amplification.

\section{Extraction of Nucleic Acids}

Nucleic acids were extracted using a Dynal ${ }^{\circledR}$ mRNA micropurification kit (Invitrogen Australia). Prior to extraction, with the samples on dry ice, $2 \mu \mathrm{L}$ (approximately $1 \mathrm{pg}$ ) pt-RNA spike and $2 \mu \mathrm{L}$ pt-DNA spike (approximately $1 \mathrm{pg}$ ) were added to each sample. Extractions were performed to the manufacturer's specifications. The lysis and wash buffers for each sample were collected and stored (combining the wash and lysis buffers). RNA was eluted from the beads using $15 \mu \mathrm{L}$ $10 \mathrm{mM}$ Tris- $\mathrm{HCl}, \mathrm{pH}$ 8.0. The genomic nucleic acids were purified from the combined lysis/wash buffers by phenol/ chloroform extraction using phase-lock gel (Eppendorf, Westbury, NY, USA) and ethanol-precipitated overnight followed by centrifugation for $45 \mathrm{~min}$ at $18,000 \times g$ in a benchtop centrifuge. Samples were washed once with $70 \%$ ethanol, centrifuged for an additional $15 \mathrm{~min}$, air-dried, and resuspended in $50 \mu \mathrm{L}$ water.

\section{Amplification of Blastocyst RNA}

Dynal-extracted mRNA was immediately used for amplification with the RiboAmp ${ }^{\circledR}$ HS RNA Amplification kit (Arcturus, Mountain View, CA, USA) with the intention to use it in future microarray experiments. Two rounds of amplification were performed on each sample as per the manufacturer's instructions.

\section{Real-Time PCR of Genomic Nucleic Acids}

Both 18S DNA and pt-DNA were quantified for each sample via quantitative PCR with an ABI PRISM ${ }^{\circledR} 7900 H T$ Sequence Detection System (Applied Biosystems). Two microliters template and $10 \mu \mathrm{L} 2 \times \mathrm{SYBR}^{\circledR}$ Green PCR master mix (Applied Biosystems) were combined with $2 \mu \mathrm{L}$ primer in a $20-\mu \mathrm{L}$ PCR mixture, and three aliquots of 5 $\mu \mathrm{L}$ were measured thereof. The primers used are listed in Table 1 along with the final primer concentration in each reaction and the annealing temperature.

\section{Real-Time PCR of cDNA}

For fibroblast RNA, cDNA was produced using SuperScript ${ }^{\mathrm{TM}}$ III

Table 1. Primers, Annealing Temperatures, and Concentration Used in PCRs

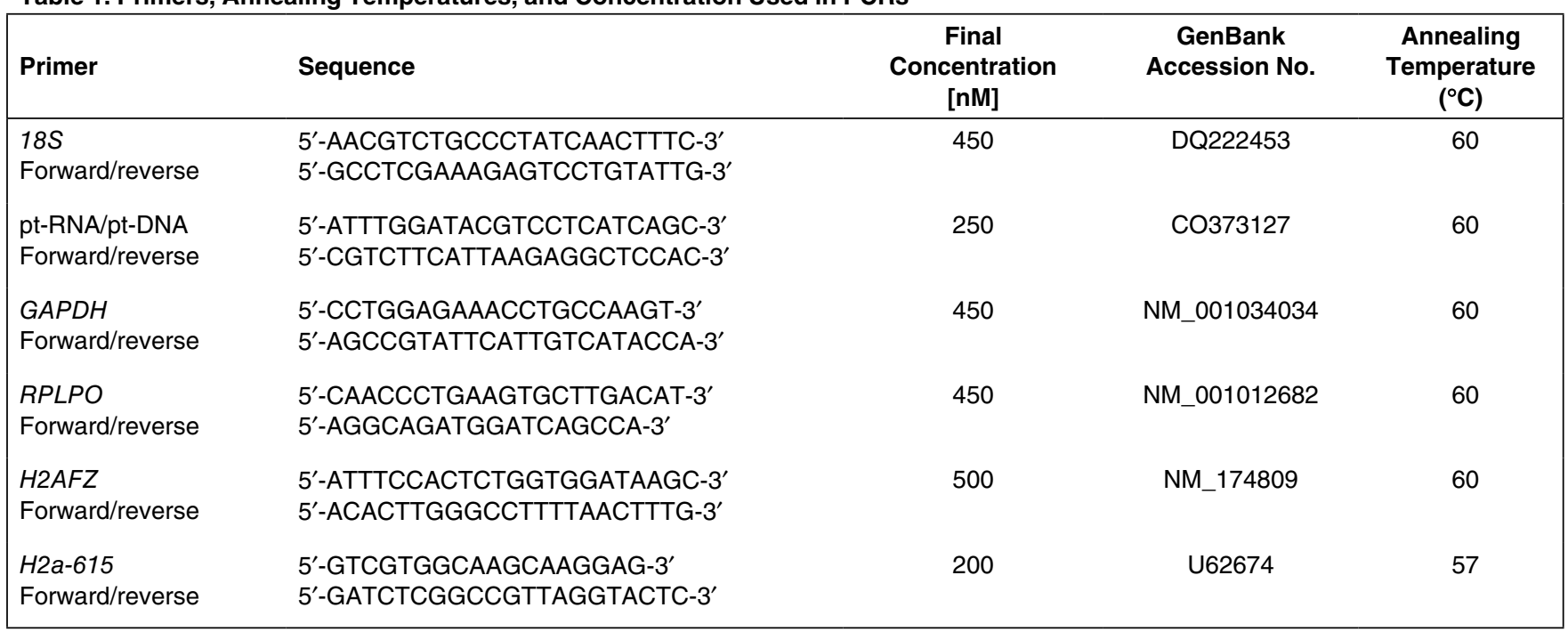




\section{Research Reports}

(Invitrogen Australia) as per the manufacturer's recommendations. The entire $15-\mu \mathrm{L}$ RNA sample was reverse transcribed in a final volume of $40 \mu \mathrm{L}$. The cDNA samples were purified using QIAquick PCR purification columns (Qiagen) as per the manufacturer's guidelines. cDNA samples were diluted 5-fold, and real-time PCR was performed with the same conditions as for the genomic nucleic acid samples. Primer sequences, annealing temperatures, and final primer concentrations for real-time PCR are listed in Table 1.

For blastocyst samples, $1 \mu \mathrm{g}$ amplified RNA was reverse transcribed using SuperScript III as per the manufacturer's recommendations. cDNA samples were diluted 5-fold, and real-time PCR was performed with the same conditions as for the genomic nucleic acid samples.

\section{Data Collection and Processing}

The URG approach is depicted in Figure 1 to visually support the stepwise calculation process presented below.

\section{Relative Cell Number Quantification}

The procedure detailed in this paper requires the quantification of cell numbers for each biological sample in order to adjust the real-time PCR data normalized to an exogenous spike and allow accurate quantification of gene expression data. The first step in this process is to measure the copies of genomic $18 \mathrm{~S}$ normalized to the pt-DNA spike. The amplification efficiency (E) of the PCRs for the 18S and pt-DNA products relative to each other were assessed through the use of a dilution series of the genomic nucleic acid samples. In all the calculations performed, the mean of the normalized expression was calculated for each sample using the QGene software (25) incorporating the use of the pt-DNA as the reference gene and the $18 \mathrm{~S}$ as the target gene (Equation 1). To avoid confusion with later calculations, the result of this calculation is termed the genome copy number (GCN).

$$
\mathrm{GCN}=\mathrm{E}_{\mathrm{pt}-\mathrm{DNA}}\left(\mathrm{pt-DNA} \mathrm{C}_{\mathrm{T}}\right) / \mathrm{E}_{18 \mathrm{~S}}\left(18 \mathrm{~S} \mathrm{C}_{\mathrm{T}}\right)
$$

[Eq. 1]

The GCN of each sample is used to adjust the data values normalized to the pt-RNA spike.

\section{Normalization for Cell Number}

Using the QGene software, the target gene can be normalized to the pt-

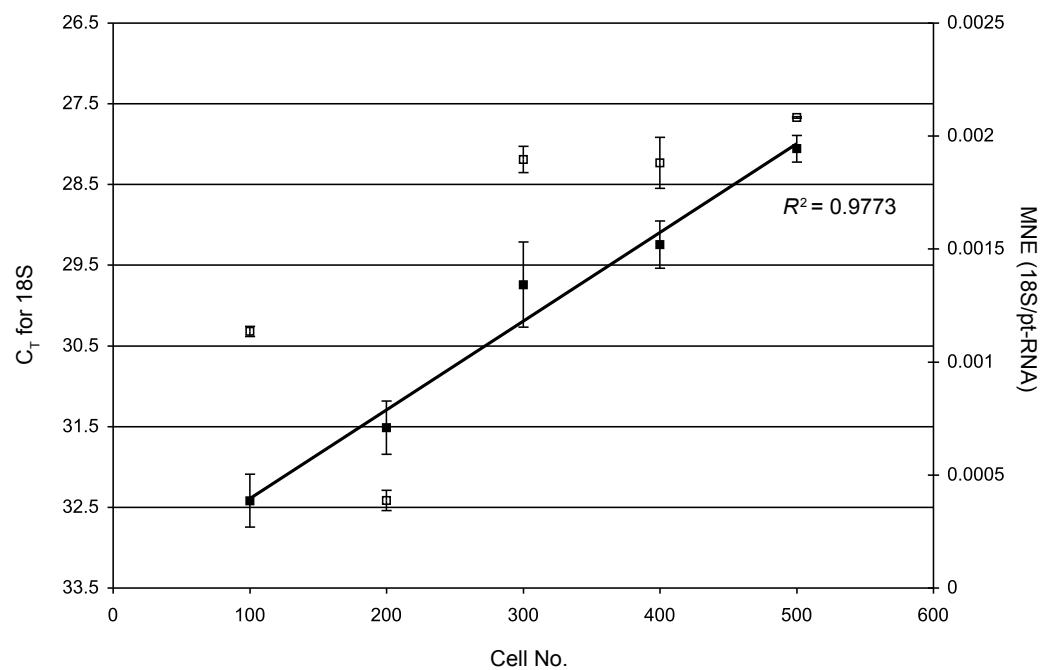

Figure 2. Plot of genome copy number (GCN) over known cell number for 18S/pt-DNA for three extractions ( $\square)$ and raw $18 \mathrm{~S}$ data $\left(\square\right.$ as $\mathbf{C}_{T}$ values). Plotting $C_{T}$ values for the 18S DNA against known cell number indicates the presence of outliers due to differences in DNA extraction efficiency. These outliers are corrected for their extraction efficiencies when the 18S DNA is normalized to the ptDNA spike. The measurement of the GCN for 18S/pt-DNA, allows for the relative cell number between samples to be calculated. pt-RNA, plant-specific transcript RNA; pt-DNA, corresponding pt-RNA PCR product, $\mathrm{C}_{\mathrm{T}}$, cycle threshold; MNE, mean normalized expression.

RNA spike. This value will be skewed by the pt-RNA spike as the amount of pt-RNA spike relative to endogenous RNA will differ from sample to sample. To allow for this, the values obtained need to be corrected for cell number by dividing by the GCN (Equation 2). This now gives a true mean normalized expression (MNE) and allows the pt-RNA spike to be used as a pseudohousekeeping gene.

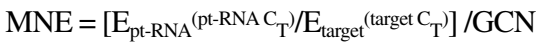

[Eq. 2]

\section{RESULTS}

\section{Validation of Cell Number Determination}

Three series of fibroblast cells were prepared with cell numbers ranging from $100-500$ cells in 100 cell increments. Before extraction, 2 $\mu \mathrm{L}$ (approximately $1 \mathrm{pg}$ ) of pt-DNA spike was added to each sample. The genomic nucleic acids were extracted, and the amount of target DNA molecules determined experimentally by quantitative PCR. Figure 2 shows both the raw $18 \mathrm{~S}$ data and the normalized $18 \mathrm{~S} / \mathrm{pt}-\mathrm{DNA}$ data as a function of the respective cell number. After plotting the $\mathrm{C}_{\mathrm{T}}$ values obtained for the 18S DNA against known cell number, outliers exist that would have a negative effect on the data if these raw measurements were used to normalize the pt-RNA spike. These outliers are corrected for their extraction efficiencies when the $18 \mathrm{~S}$ DNA is normalized to the pt-DNA spike. Error bars indicate a good reproducibility between extractions and show a good correlation $\left(R^{2}=0.9773\right)$ between the known cell number and the GCN for the 18S/pt-DNA. These results demonstrate the utility of this method for determining relative cell numbers between samples.

\section{Gene Expression Normalization in Fibroblast Cells}

To quantitatively assess the URG technique, two dilution series of a 


\section{Research Reports}

A

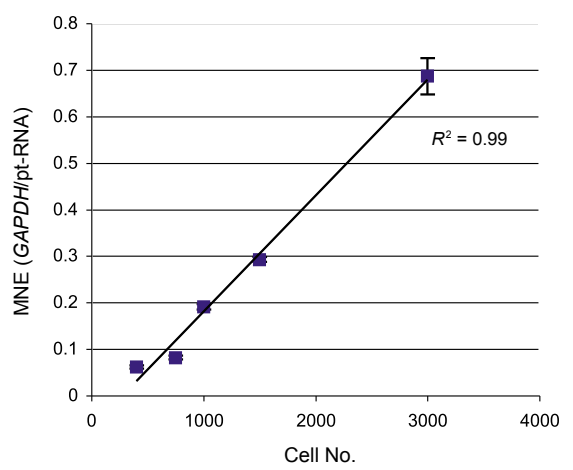

B

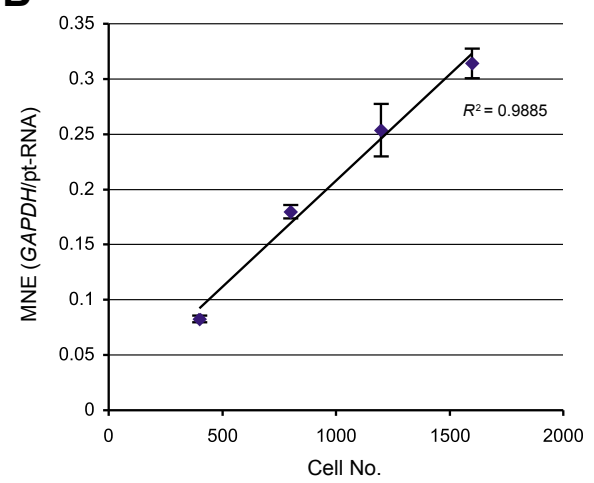

Figure 3. GAPDH expression relative to pt-RNA spike in cultured fibroblast cells from independent extraction A (panel A) and B (panel B). As the same amount of pt-RNA spike was added to each sample, the GAPDH expression increases with the number of cells extracted. pt-RNA, plant-specific transcript RNA; MNE, mean normalized expression.

cell suspension of bovine fibroblasts were prepared, each vial was frozen, and both pt-RNA and pt-DNA spikes were added prior to RNA and DNA extraction. The first dilution series consisted of cells equivalent to approximately 400, 750, 1000, 1500, and 3000 cells (extraction A). The second series consisted of six tubes with varying cell numbers with a replicate each for lowest cell count and highest cell count from approximately 400-1600 cells (extraction B). The extractions from the two dilution series were performed on separate days. QGene was used to perform all calculations for the relative quantification of GAPDH to pt-RNA spike. Real-time PCR measurement of these samples for $G A P D H$ and the pt-RNA spike are shown in Figure 3.
Amplification efficiencies of the two PCR products were calculated using a dilution series of the cDNA with the efficiency for $G A P D H$ and pt-RNA measured at 1.98 and 1.92 , respectively. In Figure 3, the GAPDH levels relative to the pt-RNA spike increase with cell number as expected. The GAPDH levels normalized to the pt-RNA spike for extraction $\mathrm{A}$, when made relative to the 400 cell sample, are 1.33, 3.1, 4.7, and 11.1 for the $750,1000,1500$, and 3000 cell samples, respectively, with an $R^{2}$ value of 0.99 . For extraction $\mathrm{B}$, the GAPDH expression normalized to the pt-RNA spike, relative to sample $400-1$ is $0.94,2.12,2.99,3.82,3.6$ for samples 400-2, 800, 1200, 1600-1, and 1600-2, respectively, with an $R^{2}$ value of 0.989 .

\section{GCN Determination and Adjustment of Target Gene Expression}

To account for the differences in cell numbers between samples, quantitative PCR was used to measure the number of copies of the $18 \mathrm{~S}$ genomic DNA and the pt-DNA spike in the fibroblast samples (above). By normalizing the $18 \mathrm{~S}$ data with the pt-DNA, the relative cell number can be determined. After calculating the GCN of 18S/pt-DNA (Equation 1), the GAPDH/pt-RNA normalized values were divided by the GCN for each sample. Figure 4 shows the data obtained for the MNE after the cell number adjustment has been made (Equation 2). The standard deviation for extractions A and B is $0.103 \pm 0.008$ and $0.0 .117 \pm 0.011$, respectively. Overall, for the two extractions, the mean is equal to 0.111 with a standard deviation of 0.012 . The GAPDH levels normalized to the pt-RNA spike for extraction $\mathrm{A}$, when made relative to the 400 cell sample, are 0.86, 0.87, 0.81, and 0.87 for the 750, 1000, 1500, and 3000 cell samples, respectively. For extraction $\mathrm{B}$, the GAPDH expression normalized to the pt-RNA spike, relative to sample $400-1$ is $0.93,1.03,1.14,1.10$, and 1.08 for samples 400-2, 800, 1200, 1600-1, and 1600-2, respectively.

\section{Gene Expression Normalization}

To compare the URG method with standard procedures, the expression
A

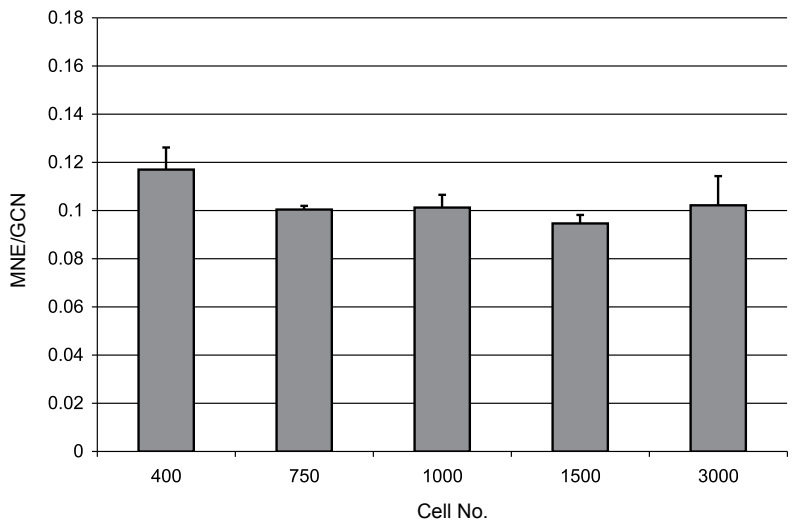

B

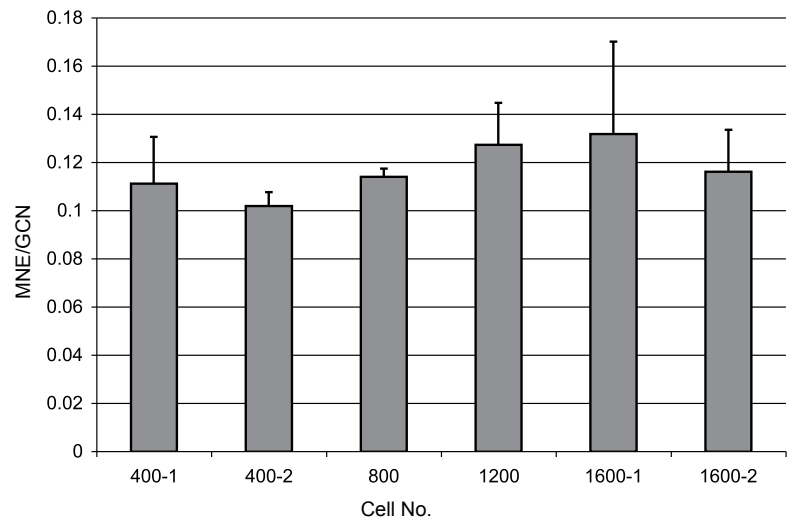

Figure 4. GAPDH expression relative to the pt-RNA spike after adjustment for fibroblast cell number (genome copy number; GCN) in extraction A (panel A) and B (panel B). The expression levels of GAPDH are now similar between samples in comparison to the non-adjusted data in Figure 2, with variation between samples likely to be due to differences in reverse transcription efficiencies between the two genes and to pipeting error. pt-RNA, plant-specific transcript RNA; MNE, mean normalized expression. 


\section{Research Reports}

A

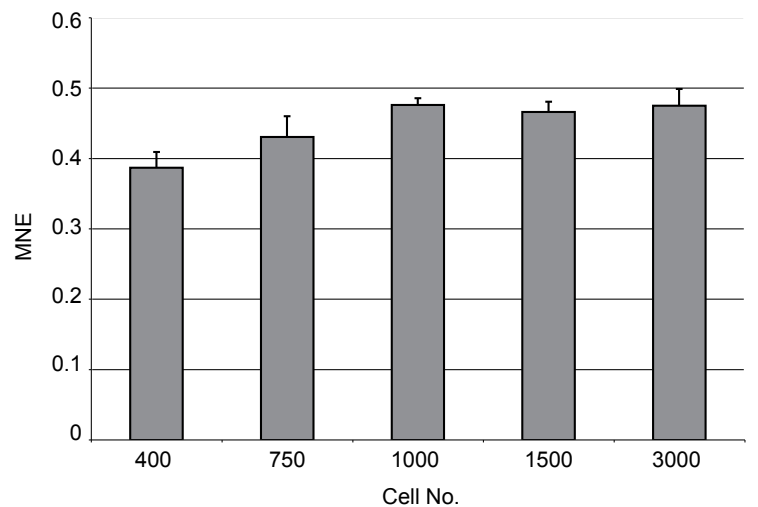

B

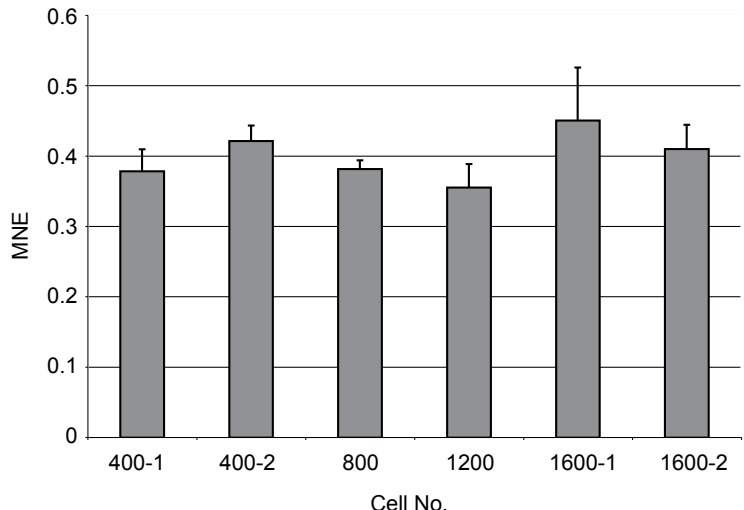

Figure 5. GAPDH normalized by RPLPO in fibroblast cells in extractions A (panel A) and B (panel B). Variation between samples is likely to be due to differences in reverse transcription efficiencies between the two genes and to pipeting error. MNE, mean normalized expression.

levels of ribosomal large protein $(R P L P O)$ and GAPDH were measured in fibroblast RNA samples, and $R P L P O$ was used to normalize the $G A P D H$ expression level. As all of the samples came from the same original cell suspension, the expression of $R P L P O$ relative to GAPDH between samples should be constant. Figure 5 shows the normalized expression levels of $G A P D H / R P L P O$ with a mean and standard deviation for extractions $\mathrm{A}$ and $\mathrm{B}$ of $0.45 \pm 0.04$ and $0.40 \pm$ 0.03 , respectively. Overall, for the two extractions, the mean is equal to 0.42 with a standard deviation of 0.042 .

After the pt-RNA spike has been adjusted for cell number, the difference in relative expression between samples is similar to that of the GAPDH/RPLPO normalization. To compare the two techniques, the coefficient of variation of the two methods was assessed. Using 10,000 bootstrap replicates, the coefficient of variation (CV) for the GAPDH/ pt-RNA normalization falls within the 95\% bootstrap confidence interval (CI) for the $\mathrm{CV}$ from the $G A P D H / R P L P O$ normalization approach (i.e., $95 \% \mathrm{CI}=$ $0.06<0.0922<0.12$ ).

\section{Comparison of Housekeeping Genes in Blastocysts}

To assess the use of the pt-RNA/ptDNA spike in SCNT (NT) and in vivo (AI) produced preimplantation embryos and to compare their stability to housekeeping genes, real-time PCR was performed on $5 \mathrm{NT}$ and 5 AI blastocysts. The GCN for the 10 blastocysts was calculated, transformed into $\mathrm{C}_{\mathrm{T}}$ values, and used to adjust the pt-RNA spike. To transform the GCN into $\mathrm{C}_{\mathrm{T}}$ values, the GCN for each sample was made relative to the GCN of one sample (the sample with the lowest GCN was used). To convert to $C_{T}$ values, the $\log$ (to the base of the PCR efficiency of the pt-RNA) of the relative GCN was calculated. This value was then subtracted from the pt-RNA $\mathrm{C}_{\mathrm{T}}$ value. The pt-RNA and genes for $R P L P O$, histone protein $H 2 A(9)$, and histone protein $\mathrm{H} 2 \mathrm{~A}$ histone family member $\mathrm{Z}(H 2 A F Z)$ were measured via real-time PCR and

Table 2. Average $C_{T}$ Values and Standard Deviations

\begin{tabular}{|lcccc|}
\hline Sample group & H2AFZ & RPLPO & pt-RNA & $\begin{array}{c}\text { Adjusted } \\
\text { pt-RNA }\end{array}$ \\
\hline Al & $23.67 \pm 0.61$ & $24.25 \pm 1.25$ & $24.73 \pm 1.23$ & $24.59 \pm 0.97$ \\
NT & $25.82 \pm 4.15$ & $25.68 \pm 2.53$ & $24.97 \pm 1.08$ & $23.15 \pm 0.90$ \\
Combined & $24.74 \pm 3.11$ & $24.82 \pm 1.96$ & $24.85 \pm 1.15$ & $23.87 \pm 1.17$ \\
$\begin{array}{l}\text { Average cycle threshold (C } \\
\text { script RNA (pt-RNA), and adjusted and standard deviations for H2AFZ, RPLPO, plant-specific tran- } \\
\text { combined (all) samples. The standard deviation for the pt-RNA is lower than for the other two genes } \\
\text { when all samples are examined. }\end{array}$ \\
\hline
\end{tabular}

their expression levels (based on $\mathrm{C}_{\mathrm{T}}$ values) between samples compared (Table 2). Expression of $H 2 A$ was undetectable in some of the NT samples (data not shown). Expression of $H 2 A F Z$ was consistent in the AI group (average $\mathrm{C}_{\mathrm{T}} \pm 0.6$ ), but was found to fluctuate between samples in the NT group (average $\left.\mathrm{C}_{\mathrm{T}} \pm 4.15\right)$. $R P L P O$ fluctuated in both $\mathrm{AI}$ (average $\mathrm{C}_{\mathrm{T}} \pm 1.25$ ) and NT groups (average $\mathrm{C}_{\mathrm{T}} \pm 2.52$ ). In contrast, the pt-RNA spike was more stable across all samples. With the adjustment for cell number, within the AI blastocysts, the standard deviation for the $\mathrm{C}_{\mathrm{T}}$ values across the samples decreased $(1.23-0.97)$ as expected. This trend was also observed in the NT embryos (1.08-0.91). When the entire set of NT and AI embryos are compared, pt-RNA measurements show less fluctuation between samples than $H 2 A F Z$ and $R P L P O$ ( \pm 1.15 versus 3.11 and 1.96 , respectively). With the adjustment for cell number, the standard deviation for the combined samples increases slightly (1.15-1.17). This suggests that there are some differences in the mRNA content between the NT and AI blastocyst samples, which could affect the ratio of pt-RNA to endogenous RNA levels.

\section{DISCUSSION}

Gene expression analysis of preimplantation embryos requires the manipulation and amplification of the RNA sample to acquire sufficient sample material to perform quantitative analysis. During the extraction 


\section{Research Reports}

and amplification procedures, there are many technical steps in which the integrity of the RNA sample can be compromised. The addition of a spike at the beginning of this process can serve as an internal control to monitor the entire process from extraction through amplification and reverse transcription. This spike has the potential to serve as a pseudo-housekeeping gene to quantify target gene expression if the relative cell number determination and normalization technique described herein is applied.

We have developed a method for measuring the relative cell number between samples using quantitative PCR techniques. The measurement of cell numbers from the dilution series gave consistent results between extractions and, when the GCN (18S/ptDNA) for the different cell numbers were compared, reflected the correct relative number of cells in each sample. The consistency of the results suggests that the relative GCN for each sample can be used as a basis to normalize for cell number, thus allowing the pt-RNA spike to be used as a reference gene. By determining the relative cell numbers between samples, the comparison of samples that have similar cell numbers and are therefore at similar developmental stages can also be confirmed.

In the experiments described here, the expression of GAPDH in fibroblast cells was normalized to the pt-RNA spike. GAPDH expression normalized to the pt-RNA spike was consistent between samples after adjusting the ptRNA spike for starting cell number. The degree of variation between samples was found to be similar to the variation obtained when two internal reference genes $(G A P D H / R P L P O)$ are used to normalize each other. The pt-RNA was more abundant than RPLPO, so normalization with the pt-RNA gives smaller values than normalization with RPLPO. As the normalization of the data drastically affects the MNE, comparison of the standard deviations between the two techniques gives misleading information. For this reason, the $\mathrm{CV}$ for the two sample sets was compared. The $\mathrm{CV}$ for the URG method falls within a $95 \%$ $\mathrm{CI}$ for the $\mathrm{CV}$ of the RPLPO normalization. Therefore, the URG method is comparable to using an internal house- keeping gene. This demonstrates that the method is a valid approach for the normalization of gene expression data in which small samples are used.

The identification of genes with stable expression across all samples in a particular experiment is vital for the assessment of gene expression. During the process of NT, reprogramming of the donor cell's nucleus from a differentiated somatic nucleus to that of a totipotent embryonic state occurs (26). As imperfect reprogramming may result in aberrant gene expression (6-8), the likelihood of finding a gene with stable expression in NT, AI, and IVF embryos is low. ACTB, a commonly used housekeeping gene, was shown to be differentially expressed in normal bovine preimplantation embryos (27). In addition, we observed unstable expression of common housekeeping genes such as $A C T B$ and GAPDH in a microarray experiment comparing the transcriptome of NT to IVF and AI bovine blastocysts (results not shown). On the other hand, histone $H 2 A$ was found to have consistent expression across the preimplantation period when the expression of a number of housekeeping genes during the development of bovine preimplantation embryos was examined (9). This is in contrast to results obtained by Bettegowda et al. (10), showing a distinct temporal expression pattern of $H 2 A$ during the bovine preimplantation development with the $H 2 A$ mRNA expression level decreasing from a maximum at the pronuclear stage to a constant low level from the 16-cell stage through to the blastocyst stage. Another study used $H 2 A$ as an endogenous reference gene to normalize and quantify gene expression in bovine NT and IVF blastocysts (28). However, while the authors have not demonstrated a stable expression pattern of $H 2 A$ in their sample set, the expression results show a considerable variation. We tested $H 2 A$ for potential use as a reference gene using the same primer sequence as described in Reference 9, but found the expression of $H 2 A$ to be undetectable in some NT samples. This gene is therefore unsuitable as a housekeeping gene in this experimental system. We also examined the expression of a variant form of the $H 2 A$ protein gene, namely $H 2 A F Z$ in NT and in vivo (AI) produced blastocysts. The expression of this gene varied considerably between the two sample groups examined. Within the AI group, the expression of $H 2 A F Z$ was stable. Expression of $H 2 A F Z$ within the NT group of blastocysts was lower (based on the average $\mathrm{C}_{\mathrm{T}}$ values) with a large variation in expression level between individual blastocysts. When the two groups were combined, there was still large variation of $\pm 3.11 \mathrm{C}_{\mathrm{T}}$ values, which equated to an almost 10fold change in expression. This gene is therefore unsuitable as a housekeeping gene in this experimental system. The URG method described in this paper entirely overcomes the problem of gene expression variation of internal reference genes, as it is independent of the transcriptome.

In conclusion, the URG method is suitable for use as a pseudo-housekeeping gene in samples where small numbers of cells are extracted. As the same amount of pt-RNA spike is added at the extraction stage and subjected to the same experimental errors as the endogenous RNA, it will serve as an indicator of extraction efficiency and integrity of each RNA sample. The pt-RNA spike can then be used as a reference gene once the adjustment for cell number has been performed. The URG method was successfully validated in fibroblast cell culture and in preimplantation embryos. It is however important to point out that it could be useful to integrate additional external RNA spikes at various concentrations into this system. This would expand on the recommendations of the External RNA Controls Consortium (ERCC) (29) and would empirically address the issue and add further confidence in normalizing the expression level of high and low abundant transcripts.

\section{ACKNOWLEDGMENTS}

N.I.B. and R.J.M. have contributed equally to this work. The authors would like to thank Dr. Walter Chang for bovine embryos, Dr. YongHong Wang for fibroblast cultures, Dr. Toni Reverter and $\mathrm{Mr}$. Wes Barris for statistical support, and the Dairy Cooperative Research Centre (CRC) for providing funding for this research. 


\section{Research Reports}

\section{COMPETING INTERESTS STATEMENT}

The authors declare no competing interests.

\section{REFERENCES}

1.Wong, M.L. and J.F. Medrano. 2005. Real-time PCR for mRNA quantitation. BioTechniques 39:75-85.

2.Karge, W.H., E.J. Schaefer, and J.M. Ordovas. 1998. Quantification of mRNA by polymerase chain reaction (PCR) using an internal standard and a nonradioactive detection method. Methods Mol. Biol. 110:43-61.

3. Thellin, O., W. Zorzi, B. Lakaye, B. De Borman, B. Coumans, G. Hennen, T. Grisar, A. Igout, and E. Heinen. 1999. Housekeeping genes as internal standards: use and limits. J. Biotechnol. 75:291-295.

4. Huggett, J., K. Dheda, S. Bustin, and A. Zumla. 2005. Real-time RT-PCR normalisation; strategies and considerations. Genes Immun. 6:279-284.

5. Dheda, K., J.F. Huggett, S.A. Bustin, M.A. Johnson, G. Rook, and A. Zumla. 2004. Validation of housekeeping genes for normalizing RNA expression in real-time PCR. BioTechniques 37:112-119.

6. Wrenzycki, C., A. Lucas-Hahn, D. Herrmann, E. Lemme, K. Korsawe, and H. Niemann. 2002. In vitro production and nuclear transfer affect dosage compensation of the X-linked gene transcripts G6PD, PGK, and Xist in preimplantation bovine embryos. Biol. Reprod. 66:127-134.

7.Pfister-Genskow, M., C. Myers, L. Childs, J.C. Lacson, T. Patterson, J. Betthauser, P.J. Goueleke, R.W. Koppang, et al. 2005. Identification of differentially expressed genes in individual bovine preimplantation embryos produced by nuclear transfer: improper reprogramming of genes required for development. Biol. Reprod. 72:546-555.

8. Somers, J., C. Smith, M. Donnison, D.N. Wells, H. Henderson, L. McLeay, and P.L. Pfeffer. 2006. Gene expression profiling of individual bovine nuclear transfer blastocysts. Reproduction 131:1073-1084.

9. Robert, C., S. McGraw, L. Massicotte, M. Pravetoni, F. Gandolfi, and M. Sirard. 2002. Quantification of housekeeping transcript levels during the development of bovine preimplantation embryos. Biol. Reprod. 67:1465-1472.

10. Bettegowda, A., O.V. Patel, J.J. Ireland, and G.W. Smith. 2006. Quantitative analysis of messenger RNA abundance for ribosomal protein L-15, cyclophilin-A, phosphoglycerokinase, $\beta$-glucuronidase, glyceraldehyde 3 -phosphate dehydrogenase, $\beta$-actin, and histone $\mathrm{H} 2 \mathrm{~A}$ during bovine oocyte maturation and early embryogenesis in vitro. Mol. Reprod. Dev. 73:267-278.

11. Eberwine, J., H. Yeh, K. Miyashiro, Y. Cao, S. Nair, R. Finnell, M. Zettel, and P. Coleman. 1992. Analysis of gene expression in single live neurons. Proc. Natl. Acad. Sci. USA 89:3010-3014.

12. Temeles, G.L., P.T. Ram, J.L. Rothstein, and R.M. Shultz. 1994. Expression patterns of novel genes during mouse preimplantation embryogenesis. Mol. Reprod. Dev. 37:121129.

13. Davis, Jr., W., P.A. De Sousa, and R.M. Schultz. 1996. Transient expression of translation initiation factor eIF-4C during the 2-cell stage of the preimplantation mouse embryo: identification by mRNA differential display and the role of DNA replication in zygotic gene activation. Dev. Biol. 174:190201.

14. De Sousa, P.A., M.E. Westhusin, and A.J. Watson. 1998. Analysis of variation in relative mRNA abundance for specific gene transcripts in single bovine oocytes and early embryos. Mol. Reprod. Dev. 49:119-130.

15. Smith, R.D., B. Brown, P. Ikonomi, and A.N. Schechter. 2003. Exogenous reference RNA for normalization of real-time quantitative PCR. BioTechniques 34:88-91.

16. Baker, P.J. and P.J. O'Shaughnessy. 2001. Expression of prostaglandin D synthetase during development in the mouse testis. Reproduction 122:553-559.

17. Macháty, Z., B.N. Day, and R.S. Prather. 1998. Development of early porcine embryos in vitro and in vivo. Biol. Reprod. 59:451455 .

18. Koo, D.-B., Y.-K. Kang, Y.-H. Choi, J.S. Park, H.-N. Kim, K.B. Oh, D.-S. Son, H. Park, et al. 2002. Aberrant allocations of inner cell mass and trophectoderm cells in bovine nuclear transfer blastocysts. Biol. Reprod. 67:487-492.

19. Kanno, J., K. Aisaki, K. Igarashi, N. Nakatsu, A. Ono, Y. Kodama, and T. Nagao. 2006. "Per cell" normalization method for mRNA measurement by quantitative PCR and microarrays. BMC Genomics 7:64.

20. Tan, S.H., A. Reverter, Y. Wang, K.A. Byrne, S.M. McWilliam, and S.A. Lehnert. 2006. Gene expression profiling of bovine invitro adipogenesis using a cDNA microarray. Funct. Integr. Genomics 6:235-249.

21. Lafri, M., C. Ponsart, M. Nibart, M. Durand, A. Morel, N. Jeanguyot, F. Badinand, K. De Mari, and P. Humblot. 2002. Influence of CIDR treatment during superovulation on embryo production and hormonal patterns in cattle. Theriogenology 58:1141-1151.

22. Hill, J.R., D.H. Schlafer, P.J. Fisher, and C.J. Davies. 2002. Abnormal expression of trophoblast major histocompatibility complex class I antigens in cloned bovine pregnancies is associated with a pronounced endometrial lymphocytic response. Biol. Reprod. 67:5563.

23. Stringfellow, D.A. and S.M. Seidel. 1998. Manual of the International Embryo Transfer Society, 3rd Ed. International Embryo Transfer Society (IETS), Savoy, IL.

24. Wells, D.N., P.M. Misica, and H.R. Tervit. 1999. Production of cloned calves follwing nuclear transfer with cultured adult granulosa cells. Biol. Reprod. 60:996-1005.
25. Muller, P.Y., H. Janovjak, A.R. Miserez, and Z. Dobbie. 2002. Processing of gene expression data generated by quantitative realtime PCR. BioTechniques 32:1372-1379.

26. Hiiragi, T. and D. Solter. 2005. Reprogramming is essential in nuclear transfer. Mol. Reprod. Dev. 70:417-421.

27. Goossens, K., M.V. Poucke, A.V. Soom, J. Vandesompele, A.V. Zeveren, and L.J. Peelman. 2005. Selection of reference genes for quantitative real-time PCR in bovine preimplantation embryos. BMC Dev. Biol. 5:2733.

28. Li, X., D. Amarnath, Y. Kato, and Y. Tsunoda. 2006. Analysis of development-related gene expression in cloned bovine blastocysts with different developmental potential. Cloning Stem Cells 8:41-50.

29. External RNA Controls Consortium. 2005 Proposed methods for testing and selecting the ERCC external RNA controls. BMC Genomics 6:150.

Received 10 July 2006; accepted 26 September 2006.

Address correspondence to Ralf Joachim Moser, CSIRO Livestock Industries, Queensland Bioscience Precinct, 306 Carmody Road, St. Lucia, QLD 4067, Australia.e-mail:ralf.moser@csiro.au

To purchase reprints of this article, contact: Reprints@BioTechniques.com 